\title{
An explicit dynamics extended finite element method. Part 2: element-by-element Stable-Explicit/Explicit dynamic scheme.
}

\author{
A. Gravouil , T. Elguedj ${ }^{1}$, H. Maigre \\ Université de Lyon, CNRS \\ INSA-Lyon, LaMCoS, UMR5259 \\ 20 avenue Albert Einstein, F69621 Villeurbanne Cedex, France
}

\begin{abstract}
This paper presents a general explicit time integration scheme for dynamics simulations using the eXtended Finite Element Method with standard critical time step. We use the generalized mass lumping technique proposed in part I of this paper. This technique allows us to consider arbitrary enrichment functions in the X-FEM for explicit dynamics simulations. In this second part, the proposed approach allows the use of standard finite elements critical time step estimates. For that purpose, we developp a classical element-by-element strategy that couples the standard central difference scheme with the unconditionally stable explicit scheme proposed by Chang (International Journal for Numerical Methods in Engineering, 77(8):11001120, 2008). This scheme coupled with X-FEM allows us to recover FE critical time steps independently of the enrichment functions considered. Furthermore, a study of the stability property of this new explicit scheme is proposed in the X-FEM framework, both with fixed enrichments and evolving enrichments with time. Some examples illustrate the good properties of the new explicit numerical time scheme and some applications to dynamics crack growth are given.
\end{abstract}

Key words: X-FEM; explicit dynamics; mass lumping; CFL condition.

\section{Introduction}

In a general point of view, explicit finite element codes are still widely used for fast transient dynamic phenomena such as crash or impact simulations. In this

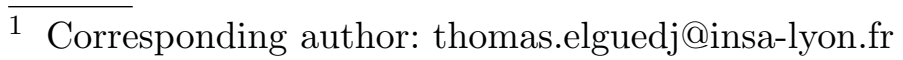


respect, explicit numerical time schemes such as the well-known central difference scheme have been widely used as they do not require numerical iterations at each time step, and also for their good properties in term of accuracy and robustness with possible nonlinearities. The main drawback of such approaches concerns their conditional stability, and in particular the Courant-FriedrichLewy (CFL) condition (see Courant et al. [14]), which involves rather small time steps in practice. However, for transient dynamic simulations (possibly non-linear), the value of the critical time step is of the order of the physical phenomena that are involved. In other words, the CFL condition is not so restrictive in practice as it corresponds to the pertinent time scale of the transient phenomenon. For transient dynamic simulations, it is also often needed to introduce in the numerical model specific interfaces, or singularities which can evolve with time (dynamic crack growth simulations, contact/impact surfaces, etc...).

In this respect, it was tried very early to couple the eXtended Finite Element Method with explicit schemes. Indeed, the ability of the X-FEM to introduce possible time evolving interface or discontinuities independently of the mesh can be very attractive for explicit transient dynamic simulations. Based on the Partition of Unity (see Babuška and Melenk [1]), the X-FEM was first applied to dynamic crack growth simulations by Belytschko and co-authors [3, 42]. In this first approach, the time scheme combines an element by element partition with an implicit time scheme for the enriched elements and an explicit time scheme for the other elements. In a next step, a general strategy for time evolving enrichments with a study of the stability properties of the Newmark time scheme family in the X-FEM framework was proposed by Réthoré et al. [34] and a general numerical time scheme family dedicated to time discontinuities has also been studied in Réthoré et al. [33]. In Réthoré et al. [34], implicit Newmark schemes have been considered, and it was shown that introducing new enrichments and preserving the old ones ensures the energy balance and stability properties of the considered time schemes in the X-FEM framework. In fact, the application of X-FEM with explicit numerical time scheme on the enriched element is rather recent. The main difficulty concerns the enrichment dependency of the critical time step both with the consistent mass matrix or standard mass lumping techniques. In other words, the critical time step of the explicit X-FEM model decreases to zero as a discontinuity or an interface tends to a node. This was first observed by Belytschko et al. [4] and overcome by using an implicit integrator for enriched elements and an explicit integrator for standard elements. Some applications of X-FEM to implicit transient dynamic crack growth simulations both for linear and nonlinear behaviour can be found in Réthoré et al. [22, 33, 34] and Prabel et al. [31] with the enrichment update strategy developed by Réthoré et al. [34] in order to ensure energy balance and stable numerical time schemes. In fact, many authors have pointed out the critical time step problem with explicit X-FEM simulations (see, e.g., [3, 32]). Recently, appropriate mass lumping techniques were proposed in order to avoid the enrichment dependency of the critical time step. 
Menouillard et al. [29, 30] developed a specific mass lumping technique for the generalized Heaviside function with application to dynamic crack growth simulations. Rozycki et al. [35] proposed a specific mass lumping technique for free boundaries and holes with constraint strain element.

In part I of this contribution, we proposed a general mass lumping technique for arbitrary enrichment functions [18]. In this respect, it becomes possible to introduce asymptotic crack front enrichments in order to avoid the mesh dependency of the crack front for tri-dimensional dynamic crack growth simulations. The main results of part I can be summarized as follows:

(1) a general X-FEM mass lumping strategy is proposed for arbitrary enrichments. It agrees with or improves the previously published mass lumping techniques in an unified manner.

(2) the new X-FEM critical time step is at least half of the standard FE critical time step without enrichments (depending on the enrichments and the finite elements considered, see part I [18]).

In this second part, we propose a new explicit X-FEM strategy in which we recover the standard FE critical time step calculated without enrichments. In other words, we are able to perform explicit X-FEM computations with arbitrary enrichments with the classical CFL condition independently of the position of the interfaces or discontinuities within the elements.

This paper is constructed as follows. In section 2, the explicit unconditionally stable algorithm proposed by Chang [11] is presented. Furthermore, the energy method is used to study its stability properties (see, e.g., $[12,21,24]$ ). In a second step, an explicit-explicit element partitioning is proposed following the previous works of Hughes and Liu [25] and Belytschko et al. [5, 37] in order to couple Chang's scheme and the central difference scheme. Then, a study of the stability property of the stable explicit-explicit scheme is proposed. Finally, its application to X-FEM with the previous mass lumping technique is developed with the following strategy: Chang's scheme is used for the enriched and blending elements, and the central difference scheme for the other elements. Some examples of dynamic crack simulations including comparisons with experiments are proposed in order to illustrate the good properties of the new explicit X-FEM time scheme, both in term of critical time step, accuracy and robustness. 


\section{An element by element stable-explicit / explicit time scheme dedicated to X-FEM}

\subsection{Enrichment independency of the critical time step and adapted mass lumping technique}

The interest of linking the X-FEM with explicit time integrators was clear from the beginning. Indeed, many transient dynamics simulations involve interfaces and discontinuities which can evolve with time. In this respect, it seems very attractive to be able not to mesh the discontinuities on a given discretization, and also to update the geometry and the mechanical properties of the interface along time with an explicit numerical time scheme. However, it was highlighted at the outset by many authors that the conditions of stability would be degraded if discontinuities are close to a node or an element boundary (see, e.g., [32]). Therefore, the global conditional stability of explicit time integrators is mainly influenced by the enriched elements in the X-FEM framework. In order to limit such a dependency, it was first proposed by various authors to built specific mass lumping techniques in order to recover a non-zero value for the critical time step when a discontinuity or an interface reaches an element boundary (see, for example, [30, 35]).

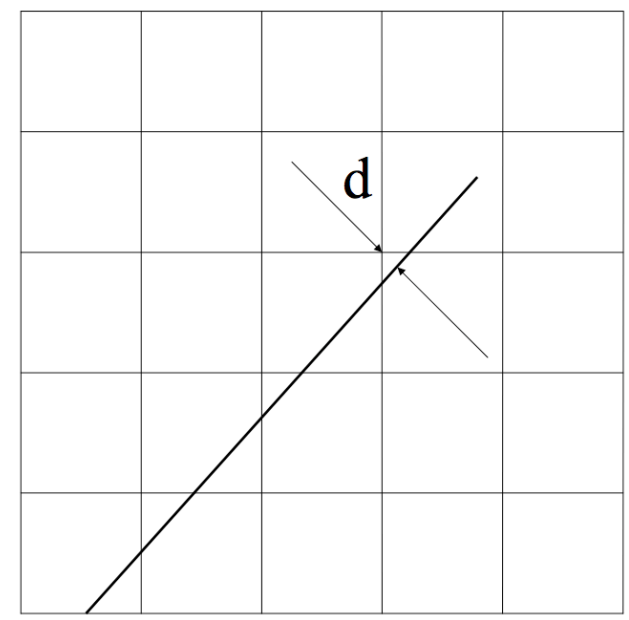

Fig. 1. Enrichment dependency of the critical time step.

It can be shown numerically that the standard mass lumping technique coupled with X-FEM involves a decreasing critical time step when $d$ tends to zero [28]. In this respect, the first step consists in building a general mass lumping technique dedicated to X-FEM which allows a non-zero value for the critical time step when an enriched interface reaches a node. In other words, it would allow us to recover an enrichment independency for the critical time step. We propose here the following notations for the X-FEM space discretization with arbitrary enrichments: 


$$
u_{i}(\mathbf{x})=\sum_{A \in \mathcal{N}} u_{i}^{A} N^{A}(\mathbf{x})+\sum_{B \in \mathcal{N}_{\text {enr }}} \sum_{\alpha=1}^{n_{e n r}} b_{\alpha i}^{B} N^{B}(\mathbf{x}) \psi_{\alpha}(\mathbf{x}),
$$

where $\mathcal{N}$ is the set of all the nodes in the mesh considered, $N^{A}(\mathbf{x})$ is the standard finite element shape function associated with node $A, u_{i}^{A}$ is the $i^{\text {th }}$ component of the associated degree of freedom, $\mathcal{N}_{\text {enr }}$ is the set of enriched nodes and $b_{\alpha i}^{B}$ is the $i^{t h}$ component of the enriched degree of freedom associated to node $B$ and to the $\alpha^{t h}$ enriched function. As a consequence, the kinetic energy of the global system at a given time step is defined as follows:

$$
T^{h}=\frac{1}{2} \dot{U}_{x-f e m}^{T} M_{L} \dot{U}_{x-f e m},
$$

with

$$
M_{L}=\sum_{e}\left(m_{L \text { enr }}^{e}+m_{L \text { std }}^{e}\right)
$$

where $M_{L}$ is the sum of the lumped mass matrices of each finite element, and $\dot{U}$ the discretized velocity field with X-FEM enrichments. In this respect, it involves a mass matrix larger than the corresponding mass matrix without enrichment. From this definition, it has been shown in part I [18] that the following mass lumping formula:

$$
\left[m_{L \text { enr }}^{e}\right]_{i j}=\delta_{i j} m_{L} \quad \text { with } \quad m_{L}=\frac{1}{\sum_{i=1}^{\text {nnode }} \psi^{2}\left(x_{i}\right)} \int_{\Omega_{e}} \rho \psi^{2} d \Omega_{e},
$$

allows for arbitrary enrichment functions with non zeroed value of the critical time step. This critical time step is smaller in practice than the standard critical time step obtained without enrichment:

$$
0<\Delta t_{c}^{x-f e m} \leq \Delta t_{c}^{f e m}
$$

with a minimal ratio between $\Delta t_{c}^{x-f e m}$ and $\Delta t_{c}^{f e m}$ of the order of $1 / 2$. In the aim to recover the following property:

$$
\Delta t_{c}^{x-f e m} \equiv \Delta t_{c}^{f e m}
$$

we propose here an explicit / explicit coupling technique which uses the explicit unconditionally stable time scheme presented in the next section.

\subsection{Presentation of an unconditionally stable explicit time scheme with an energetic stability study}

From the pioneered works in applying explicit time numerical schemes to transient dynamic finite element simulations (see, e.g., $[2,6,27]$ ), many authors have tried to improve explicit time schemes properties with respect to balance 
equations (momentum, moment of momentum, energy, ...), order of accuracy, period distorsion and amplitude error. Generalizations of explicit strategies were also proposed, such as subcycling algorithms (see for example Smolinski et al. [37, 38] and Daniel [15]), mixed multi-time algorithms (see Belytschko et al. [5, 7, 8] and Sotelino [39]), or dual mixed multi-time algorithms (see Gravouil and Combescure [12, 21]), both for linear and nonlinear applications. However, trully unconditionally stable explicit time schemes have only been proposed recently by Chang $[9,10]$ and Tamma et al. [36, 40]. Such explicit time integrators have very attractive properties as they allow time steps greater than the standard critical time step. However, in practice, due to the high frequency phenomena which always occur in transient dynamic problems, one order of magnitude greater than the standard critical time is sufficient to obtain an accurate modelling. Some extensions of these explicit time schemes are also applied to elastic-plastic behaviour, or general non-linear behaviour considered in structural dynamics by Zhou et al. [41] and Chang [11].

In this respect, we present here the unconditionally stable explicit time scheme proposed by Chang which can be summarized as follows for linear elastic behaviour without physical damping:

$$
\begin{gathered}
M \ddot{U}_{n+1}+K U_{n+1}=F_{n+1}, \\
U_{n+1}=U_{n}+\beta_{1}^{-1} \Delta t \dot{U}_{n}+\beta_{2}^{-1} \Delta t^{2} \ddot{U}_{n}, \\
\dot{U}_{n+1}=\dot{U}_{n}+\frac{1}{2} \Delta t\left(\ddot{U}_{n}+\ddot{U}_{n+1}\right),
\end{gathered}
$$

with

$$
\begin{gathered}
\beta_{1}=\left(I+\frac{1}{4} \Delta t^{2} M^{-1} K\right), \\
\beta_{2}^{-1}=\frac{1}{2} \beta_{1}^{-1},
\end{gathered}
$$

where $U, \dot{U}, \ddot{U}$ correspond respectively to the discretized displacement, velocity and acceleration fields at time $t_{n}$ or $t_{n+1}, \mathrm{M}$ and $\mathrm{K}$ the consistent mass and stiffness matrices.

This numerical time scheme can be considered as an explicit one by the fact that the displacement field can be calculated with Eq. (7b) before the use of the equilibrium Eq. (7a). In a second step, the acceleration field is calculated with Eq. (7a), and finally the velocity field with Eq. (7c). It can be noticed that the displacement field calculation implies the use of two constant operators $\beta_{1}$ and $\beta_{2}$. This involves to define a preliminary linear system for the displacement field calculation. This is the main difference with standard explicit time schemes which do not need the use of linear system solving (except with the use of Lagrange multipliers). Chang's unconditionally stable explicit time scheme has already been studied in detail in several papers by Chang 
[9-11], both in term of unconditional stability (amplification matrix), second order accuracy, absence of numerical dissipation, and overshooting effect in displacement and in velocity (see, e.g., $[20,23]$ ). We propose here to recall its stability properties by the use of an energy approach. We introduce the following notations:

$$
[X]=X_{n+1}-X_{n} \text { and }<X>=\frac{1}{2}\left(X_{n+1}+X_{n}\right) .
$$

As it is commonly done (see Hughes [24]) we assume absence of external loads for this stability study. From the difference of equilibrium equations at time $t_{n}$ and $t_{n+1}$, multiplied by the quantity $[\dot{U}]$, we obtain:

$$
[\dot{U}]^{T} M[\ddot{U}]+[\dot{U}]^{T} K[U]=0 .
$$

From Eqs. (7a) to (7c), we deduce the following relations:

$$
\beta_{1}[U]=\Delta t\left(\dot{U}_{n}+\frac{1}{2} \Delta t \ddot{U}_{n}\right) \quad \text { and } \quad[\dot{U}]=\Delta t<\ddot{U}>
$$

which can be combined as follows:

$$
\beta_{1}[U]=\Delta t\left(<\dot{U}>-\frac{1}{4} \Delta t[\ddot{U}]\right) .
$$

From the definition of $\beta_{1}$ given in Eq. (8a), we can write:

$$
[U]=\Delta t\left(<\dot{U}>-\frac{1}{4} \Delta t[\ddot{U}]\right)-\frac{1}{4} \Delta t^{2} M^{-1} K[U] .
$$

The difference of equilibrium equations at time $t_{n+1}$ and time $t_{n}$ leads to:

$$
K[U]=-M[\ddot{U}] .
$$

Combining Eqs. (12) and (14) we obtain:

$$
[U]=\Delta t<\dot{U}>\text {. }
$$

In a last step, the combination of Eqs. (10), (11) and (15), leads to:

$$
<\ddot{U}>^{T} M[\ddot{U}]+<\dot{U}>^{T} K[\dot{U}]=0 .
$$

Following the methodology of Hughes [24], it corresponds here to a sum of quadratic terms without numerical dissipation term. Furthermore, Eq. (16) is identical to the one obtained for the unconditionally stable constant average acceleration method (Newmark time scheme with $\beta=0.25$ and $\gamma=0.5$ ). This confirms with an energetic approach that Chang's explicit time scheme is also unconditionally stable. 
As a conclusion, it can be retained that the general properties of Chang's unconditionally stable explicit time scheme are exactly the same as those for the constant average acceleration method, in an explicit framework.

\subsection{Development of a stable-explicit / explicit strategy}

In order to couple the central difference method and Chang's explicit time scheme, we propose to follow the element by element strategy developed by Hughes and Liu [25]. We briefly recall here the original method applied to implicit-explicit predictor-corrector strategy for Newmark time schemes:

$$
\begin{array}{r}
M \ddot{U}_{n+1}+K \tilde{U}_{n+1}=F_{n+1}, \\
\tilde{U}_{n+1}=U_{n}+\Delta t \dot{U}_{n}+\Delta t^{2}\left(\frac{1}{2}-\beta\right) \ddot{U}_{n}, \\
\tilde{\dot{U}}_{n+1}=\dot{U}_{n}+\Delta t(1-\gamma) \ddot{U}_{n}, \\
U_{n+1}=\tilde{U}_{n+1}+\Delta t^{2} \beta \ddot{U}_{n+1}, \\
\dot{U}_{n+1}=\tilde{\dot{U}}_{n+1}+\Delta t \gamma \ddot{U}_{n+1} .
\end{array}
$$

In a second step, it consists in a partition of implicit elements and explicit elements:

$$
\begin{array}{r}
M \ddot{U}_{n+1}+K^{E} \tilde{U}_{n+1}+K^{I} U_{n+1}=F_{n+1}, \\
\tilde{U}_{n+1}=U_{n}+\Delta t \dot{U}_{n}+\Delta t^{2}\left(\frac{1}{2}-\beta\right) \ddot{U}_{n}, \\
\tilde{\dot{U}}_{n+1}=\dot{U}_{n}+\Delta t(1-\gamma) \ddot{U}_{n}, \\
U_{n+1}=\tilde{U}_{n+1}+\Delta t^{2} \beta \ddot{U}_{n+1}, \\
\dot{U}_{n+1}=\tilde{\dot{U}}_{n+1}+\Delta t \gamma \ddot{U}_{n+1},
\end{array}
$$

where

$$
M=M^{I}+M^{E} \text { and } K=K^{I}+K^{E} \text { and } F=F^{I}+F^{E} .
$$

In Eq. (19), $M^{I}$ is a consistant mass matrix linked to the implicit part, and $M^{E}$ is a lumped mass matrix linked to the explicit part. In the same way, we propose to develop a stable explicit - explicit time scheme according to 
Eqs. (18a) to (18e):

$$
\begin{array}{r}
M \ddot{U}_{n+1}+K U_{n+1}=F_{n+1}, \\
U_{n+1}^{E}=U_{n}^{E}+\Delta t \dot{U}_{n}^{E}+\frac{\Delta t^{2}}{2} \ddot{U}_{n}^{E}, \\
U_{n+1}^{E S}=U_{n}^{E S}+\beta_{1}^{-1} \Delta t \dot{U}_{n}^{E S}+\beta_{1}^{-1} \frac{\Delta t^{2}}{2} \ddot{U}_{n}^{E S}, \\
\dot{U}_{n+1}=\dot{U}_{n}+\frac{\Delta t}{2}\left(\ddot{U}_{n}+\ddot{U}_{n+1}\right) .
\end{array}
$$

with the following definitions:

$$
\begin{gathered}
M=M^{S E}+M^{E} \text { and } K=K^{S E}+K^{E} \text { and } F=F^{S E}+F^{E}, \\
\beta_{1}=\left(I+\frac{1}{4} \Delta t^{2}\left(M^{S E}\right)^{-1} K^{S E}\right),
\end{gathered}
$$

where upperscripts $S E$ and $E$ correspond respectively to Chang's stable explicit time scheme and the central difference time scheme. Following the strategy of Hughes [24], one can also study the global stability properties of the proposed stable-explicit/explicit method by the use of an energetic approach.

Remark 1 It should be noted here, as presented previously, that the numerical cost of Chang's scheme is bigger than the one of the central difference time scheme. It involves the assembly and inverse computation of the $\beta_{1}$ operator at the beginning of the time loop. Once $\beta_{1}^{-1}$ is formed, a matrix vector computation is needed at each time step to form the new displacement vector as can be seen in Eq. (7b). The main purpose of creating an element by element scheme is to limit the size of the SE group so that this additional cost is kept as low as possible.

In order to obtain the stability conditions for the stable-explicit/explicit algorithm, one first recalls the energy method applied to the Newmark algorithm alone without mechanical damping (see, e.g., Hughes [24]):

$$
\ddot{U}_{n+1}^{T} A \ddot{U}_{n+1}+\dot{U}_{n+1}^{T} K \dot{U}_{n+1}=\ddot{U}_{n}^{T} A \ddot{U}_{n}+\dot{U}_{n}^{T} K \dot{U}_{n}-(2 \gamma-1)\left[\ddot{U}_{n}\right]^{T} A\left[\ddot{U}_{n}\right]
$$

where

$$
A=M+\Delta t^{2}(\beta-\gamma / 2) K .
$$

To establish the stability conditions, we only need to determine when $A$ is positive-definite (see, e.g., [21]). For the Newmark time scheme, the standard stability properties are obtained:

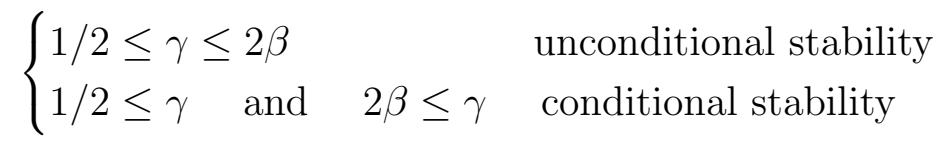

Furthermore, the energy method has already been applied to implicit-explicit algorithms with an element-by-element strategy in order to study its global 
stability properties by Hughes and Liu [25]. In the same way, we obtain the following expression for the stable explicit - explicit partition:

$$
\begin{aligned}
\ddot{U}_{n+1}^{T}\left(A^{S E}+A^{E}\right) \ddot{U}_{n+1}+\dot{U}_{n+1}^{T} K \dot{U}_{n+1} & =\ddot{U}_{n}^{T}\left(A^{S E}+A^{E}\right) \ddot{U}_{n}+\dot{U}_{n}^{T} K \dot{U}_{n} \\
& -(2 \gamma-1)\left[\ddot{U}_{n}\right]^{T}\left(A^{S E}+A^{E}\right)\left[\ddot{U}_{n}\right]
\end{aligned}
$$

where

$$
\begin{gathered}
A^{S E}=M^{S E}+\Delta t^{2}(\beta-\gamma / 2) K^{S E}, \\
A^{E}=M^{E}+\Delta t^{2}(\beta-\gamma / 2) K^{E} .
\end{gathered}
$$

Again, the proof is identical to that of the first theorem with $A^{E S}+A^{E}$ in place of $A$ with $\beta=0$ and $\gamma=1 / 2$ (see, e.g., [25]). In other words, the global stability is preserved according to the stability properties of each partition. In this respect, we have shown in the previous section that the stability property of the stable explicit partition is identical to the constant average acceleration method. Furthermore, if the same global time step is considered for each partition, no numerical dissipation is introduced at the interface between the partitions.

As a conclusion, we propose here to combine in an element by element partitioning an unconditionally stable explicit time scheme with the central difference method. In the next section, we use this strategy in an explicit X-FEM framework with the proposed specific lumped mass matrix for the stable explicit partition and a standard lumped mass matrix for the central difference partition.

\subsection{An explicit X-FEM method with a standard critical time step}

The main goal of this paper is to propose an explicit X-FEM method with:

(1) a specific mass lumping technique for arbitrary enrichments

(2) an explicit method with standard critical time step

In this section, we propose to reach the second goal by the use of an unconditionally stable explicit time scheme for the enriched elements and the blending elements, with the central difference method for the other ones. In this respect, we can apply the element by element partitioning proposed in section 2.3. This choice for the partitioning is what ensures us to have a numerical cost due to the use of Chang's scheme as low as possible. We only put in the SE group the elements for which the critical time step is too small, that is the enriched ones. In most cases, as will be seen on the examples this ensures us that the 
size of the SE problem is of one dimension lower, that is in 2D, it has the size of a $1 \mathrm{D}$ problem.

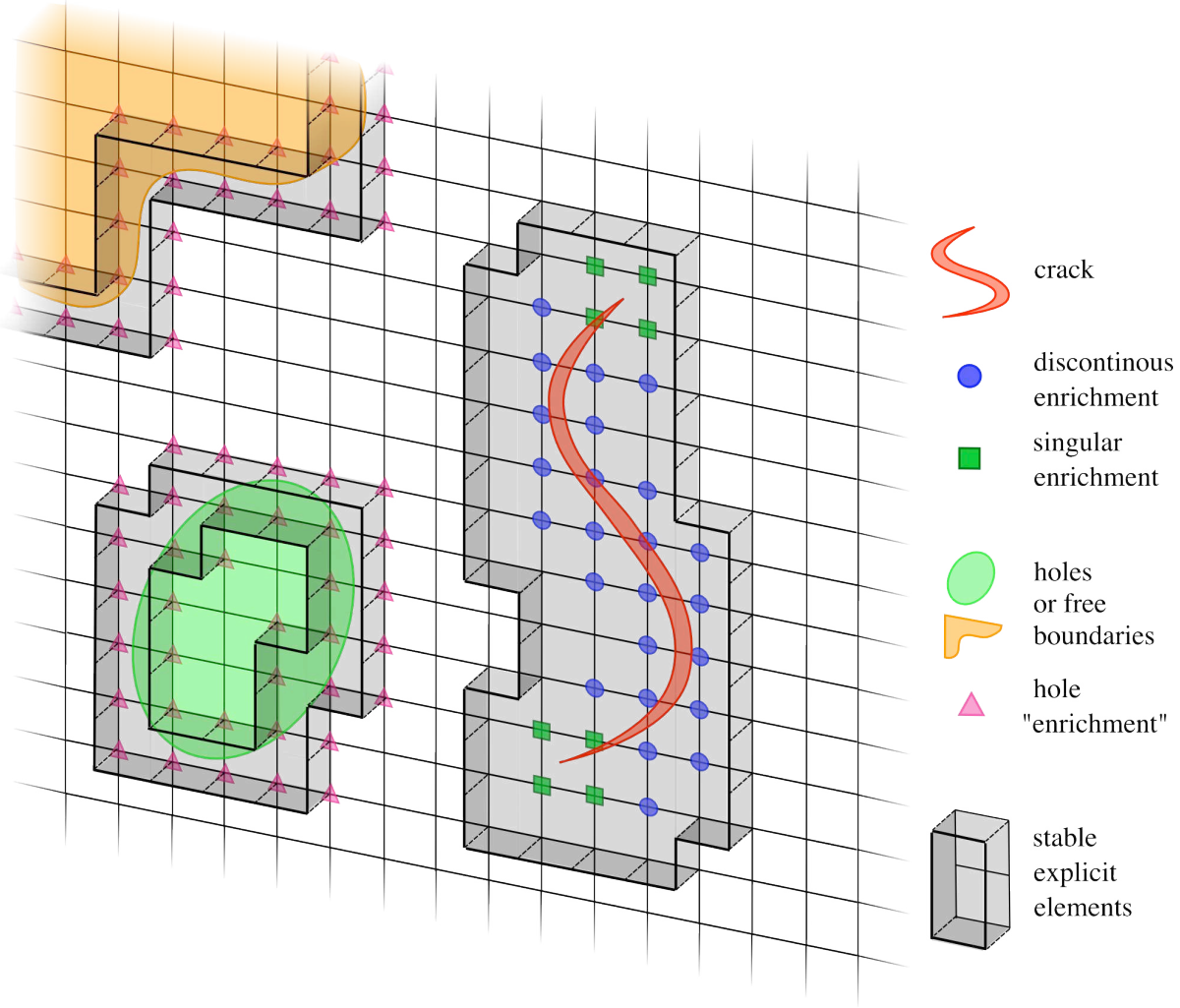

Fig. 2. Stable-Explicit / Explicit element partitionning. Note that the elements completely contained inside a hole are deleted.

In Figure 2, we illustrate the application of such a partitioning with different kinds of fixed enrichments (crack, hole, free boundary, ...). The global stability of explicit element by element partitioning dedicated to X-FEM can be proved in the same way as section 2-2. Indeed, the same energetic expression can be obtained with standard and enriched degrees of freedom. However, the main interest of X-FEM resides in its ability to model evolving interfaces with time. In this respect, it has been shown that introducing new enrichments and preserving the old ones ensure the energy balance and the global stability of the considered time scheme (see, e.g., $[13,33,34]$ and part I, paragraph 2.2.3.1). This strategy can also be extended to the proposed stable explicitexplicit partitioning.

For the case of evolving enrichment, a prime example being crack growth simulation, the partitioning evolves with the discontinuity. As the discontinuity moves from one element to another, new enriched elements are added in the SE group as presented in Figure 3. This has various implications on the matrices of the system. First, mass and stiffness matrices of the SE group are getting bigger because of the new enrichements that are created and because of new elements that are added in the group. Second, as some elements are changing 


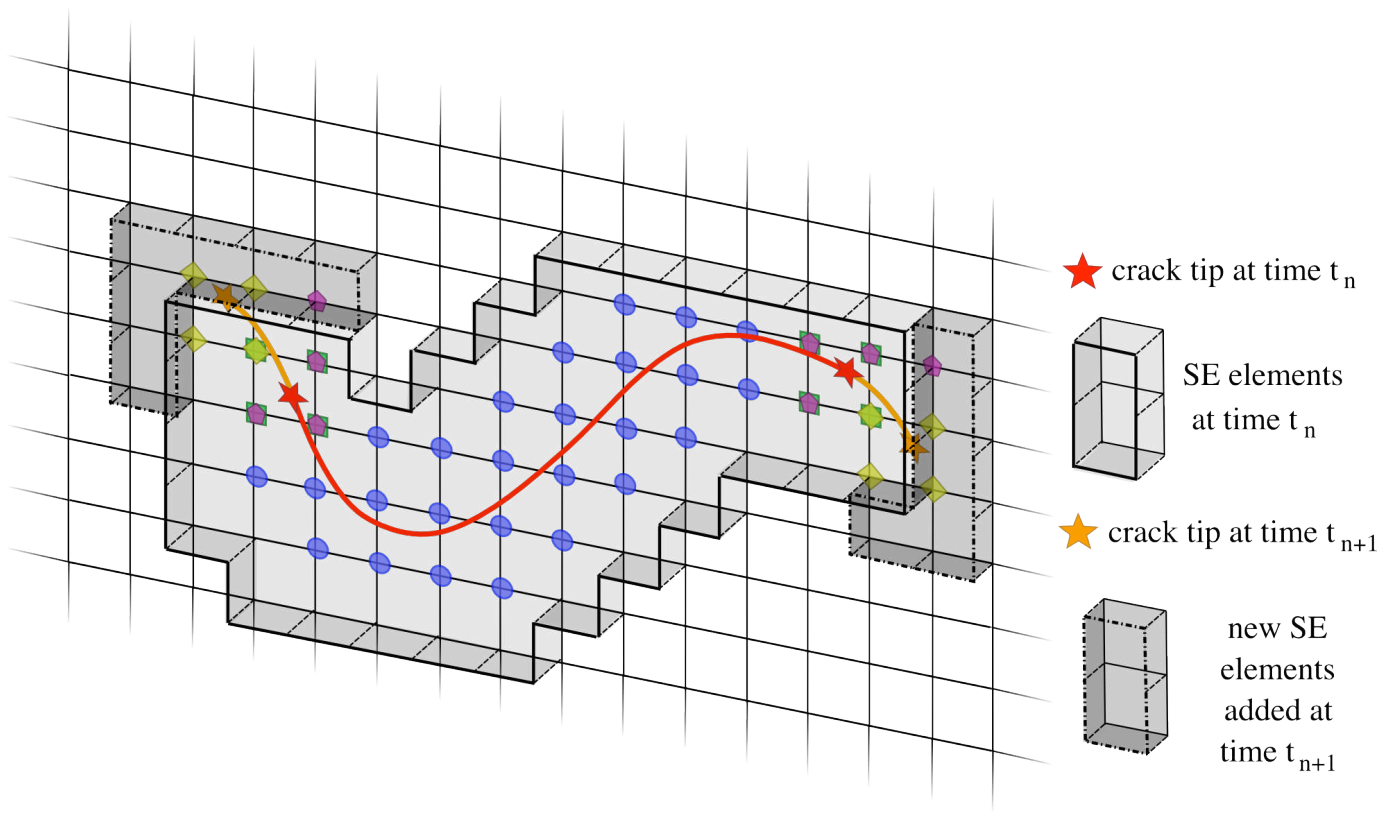

Fig. 3. Stable-Explicit / Explicit element partitioning update for a propagating crack.

from the E group to the SE group, the mass and stiffness matrices of the $\mathrm{E}$ group are getting smaller. Finally, the $\beta_{1}$ operator of the stable scheme is also getting bigger and must be recomputed. The numerical cost of this operation is what motivates the use of an element-by-element scheme with the proposed partitioning. The size of the SE group is maintained as small as possible and its growth is maintained as low as possible.

\section{$3 \quad$ Numerical Examples}

All the examples presented in the following are treated under plane strain conditions. Furthermore, we apply here the general X-FEM stable explicitexplicit strategy to dynamic fracture mechanics. In such a case, we consider the following discontinuous and asymptotic enrichments:

$$
H(x)= \begin{cases}+1 & \text { if } x \geq 0 \\ -1 & \text { if } x<0\end{cases}
$$

and

$$
\left[B_{\alpha}\right]=\sqrt{r} \sin \frac{\theta}{2}
$$

in order to capture respectively the discontinuity along the crack faces, and the asymptotic behavior close to the crack tip. As shown by different authors, 
a sufficient accuracy is obtained with static linear elastic crack tip enrichments given in Eq. (30) for dynamic crack growth simulations in mixed modes (see, e.g., $[22,34]$ ) and it was observed in Part 1 that using only the first asymptotic enrichment function provides very accurate results thus reducing the cost of the method.

\subsection{Stationary and moving semi-infinite mode I crack}

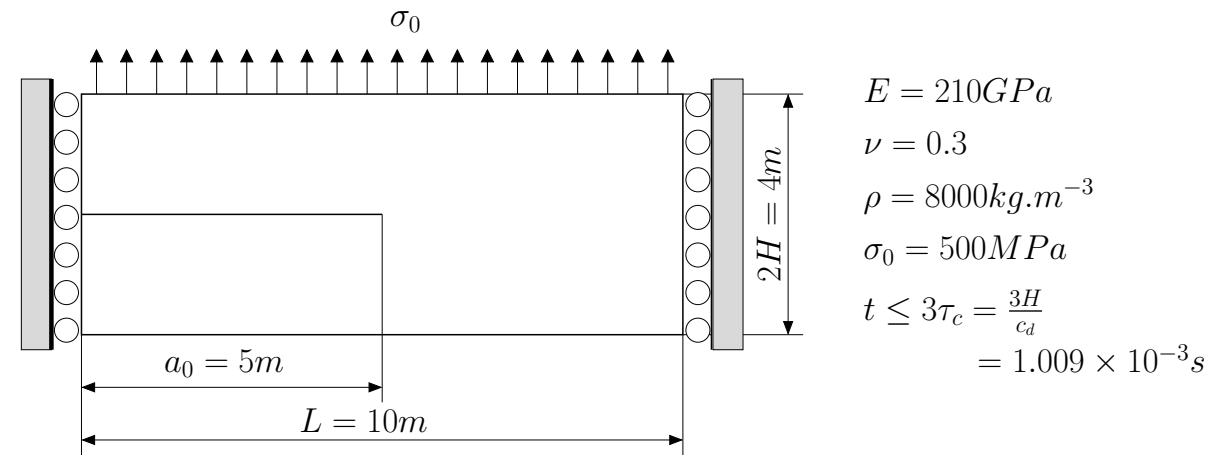

Fig. 4. Mode I semi-infinite crack problem: finite geometry and material parameters.

We consider as a first example the case of a semi-infinite mode I crack in an infinite medium submitted to a tensile stress wave. The analytical solution for the mode I dynamic stress intensity factor was obtained by Freund [19]. We consider the finite geometry given in Figure 4, therefore we can only compare the results with the analytical solution until the tensile stress wave is reflected on the bottom side and reaches again the crack tip. The time needed by the stress wave to reach the crack tip for the first time is $\tau_{c}=\frac{H}{c_{d}}$, where $c_{d}$ is the dilatational wave speed. The total simulation is therefore limited to $t \leq 3 \tau_{c}=1.009 \times 10^{-3} s$ with the material parameters given in Figure 4 .

As the wave reaches the crack tip, the mode I dynamic stress intensity factor for a stationary crack is given by:

$$
K_{I}^{d y n}(0, t)=\frac{2 \sigma_{0}}{1-\mu} \sqrt{\frac{c_{d} t(1-2 \mu)}{\pi}}
$$

where $\mu$ is the second Lamé constant. For a moving crack tip, the mode I dynamic stress intensity factor is given by:

$$
K_{I}^{d y n}(\dot{a}, t)=k(\dot{a}) K_{I}^{d y n}(0, t)
$$

where $k$ is a universal function of the crack tip speed $\dot{a} . k$ can be approximated by the following expression:

$$
k(\dot{a})=\frac{1-\dot{a} / c_{r}}{1-\dot{a} / 2 c_{r}},
$$




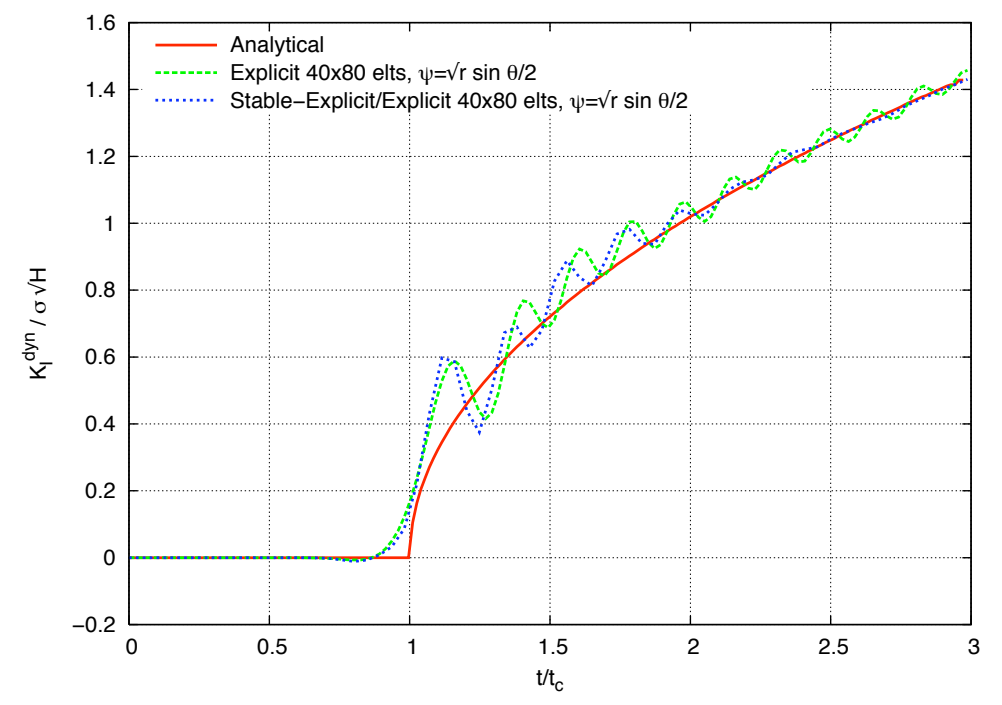

Fig. 5. Normalized mode I stress intensity factor vs. normalized time for a stationary semi-infinite crack: analytical solution, $40 \times 80$ elements mesh with central difference explicit scheme and time step rule of Part $1\left(\Delta t=\Delta t_{c} / 2\right), 40 \times 80$ elements mesh with element-by-element explicit scheme $\left(\Delta t=\Delta t_{c}\right)$.

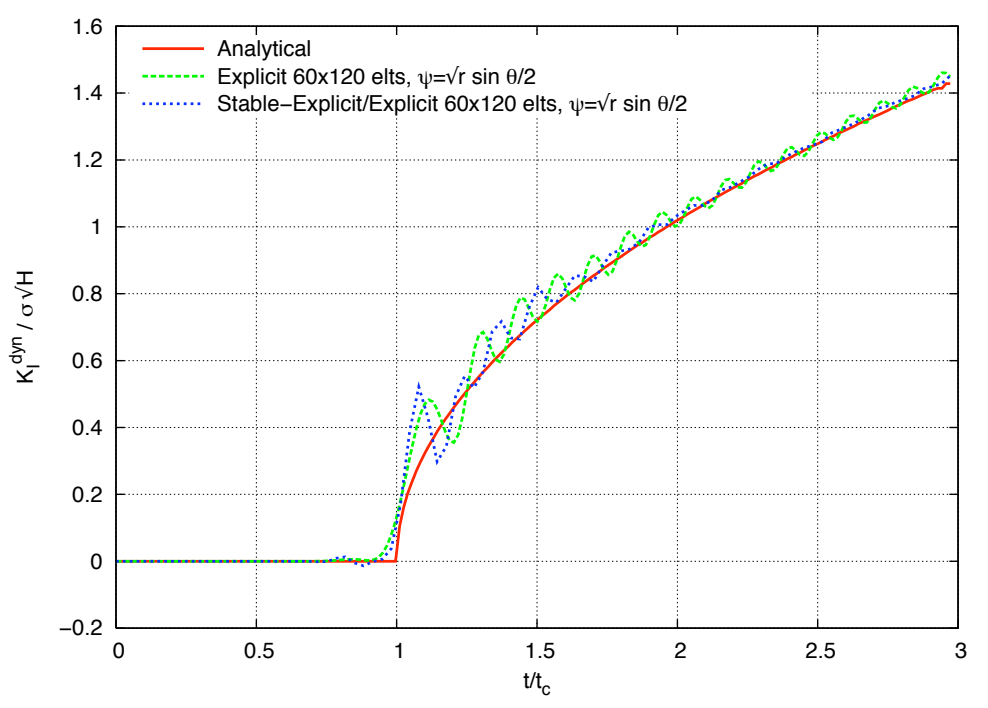

Fig. 6. Normalized mode I stress intensity factor vs. normalized time for a stationary semi-infinite crack: analytical solution, $60 \times 120$ elements mesh with central difference explicit scheme and time step rule of Part $1\left(\Delta t=\Delta t_{c} / 2\right), 60 \times 120$ elements mesh with element-by-element explicit scheme $\left(\Delta t=\Delta t_{c}\right)$.

where $c_{r}$ is the Rayleigh wave speed. Finally, one can write:

$$
K_{I}^{d y n}(\dot{a}, t)=\frac{2 \sigma_{0}}{1-\mu} \sqrt{\frac{c_{d} t(1-2 \mu)}{\pi}} \frac{1-\dot{a} / c_{r}}{1-\dot{a} / 2 c_{r}} .
$$

We first use a mesh of $40 \times 80$ quadrilateral elements with standard explicit central difference scheme with a critical time step of $\Delta t_{c}^{x-f e m}=\Delta t_{c}^{f e m} / 2=7.5 \mu s$ 
(that is a simulation of 134 time steps) and the proposed stable-explicit / explicit scheme with a critical time step of $\Delta t_{c}^{x-f e m}=\Delta t_{c}^{f e m}=15 \mu \mathrm{s}$ (that is a simulation of 67 time steps). We compare the normalized mode I dynamic stress intensity factor $K_{I}^{d y n} / \sigma_{0} \sqrt{H}$ as a function of the normalized time $t / \tau_{c}$ obtained with and without the proposed dynamic scheme and with the analytical solution.

The result can be seen in Figure 5. We can observe that all the results are close to the analytical solution. The solution with a standard explicit central difference time integrator presents some oscillations whereas the solution with the proposed time integration scheme presents attenuating oscillations. Around a time of $t \simeq 2 \tau_{c}$ the result presents almost no oscillations and is identical to the analytical solution. This demonstrates that for a mode I stationary crack the proposed time integration scheme eliminates some of the oscillations that are obtained when the critical time step for the whole mesh is limited by the one of the enriched elements.

The same calculation was also performed with a mesh of $60 \times 120$ quadrilateral elements with a standard explicit central difference scheme with a critical time step of $\Delta t_{c}^{x-f e m}=5 \mu s \simeq \Delta t_{c}^{f e m} / 2$ (that is a simulation of 200 time steps) and the proposed Stable-Explicit/Explicit scheme with a critical time step of $\Delta t_{c}^{x-f e m}=\Delta t_{c}^{f e m}=10.99 \mu \mathrm{s}$ (that is a simulation of 91 time steps). In Figure 6, we can observe that both cases fit quite well the analytical solution but that again the element-by-element scheme presents fewer oscillations and that these oscillations are not seen for a time $t>2 \tau_{c}$.

As in various references $[3,4,17,29,33,34]$, we now consider the case of stationary then moving crack. The crack remains stationary for $0 \leq t<1.5 \tau_{c}$ then propagates in mode I at a constant velocity $\dot{a}=1500 \mathrm{~m} . \mathrm{s}^{-1}$ for $t \geq 1.5 \tau_{c}$.

We consider the same two cases as in the previous example. In Figure 7, we compare the results obtained with a mesh composed of $40 \times 80$ quadrilateral elements with standard explicit central difference scheme with a critical time step of $\Delta t_{c}^{x-f e m}=\Delta t_{c}^{f e m} / 2=7.5 \mu s$ (that is a simulation of 134 time steps) and the proposed Stable-Explicit/Explicit scheme with a critical time step of $\Delta t_{c}^{x-f e m}=\Delta t_{c}^{f e m}=15 \mu \mathrm{s}$ (that is a simulation of 67 time steps). The results compare relatively well with the analytical solution although they both presents oscillations when the crack is moving as it is the case with other XFEM explicit dynamics techniques presented in Belytschko et al. [4], $\mathrm{Zi}$ et al. [42] and Menouillard et al. [29]. It is interesting to note here, that we did not apply any numerical filter to the results obtained with the proposed method contrary to the ones presented in the References cited above. We can observe that the results obtained with the proposed method presents fewer oscillations with a lower amplitude compared to the results obtained with a standard central difference scheme and the time step rule of Part 1 . This 


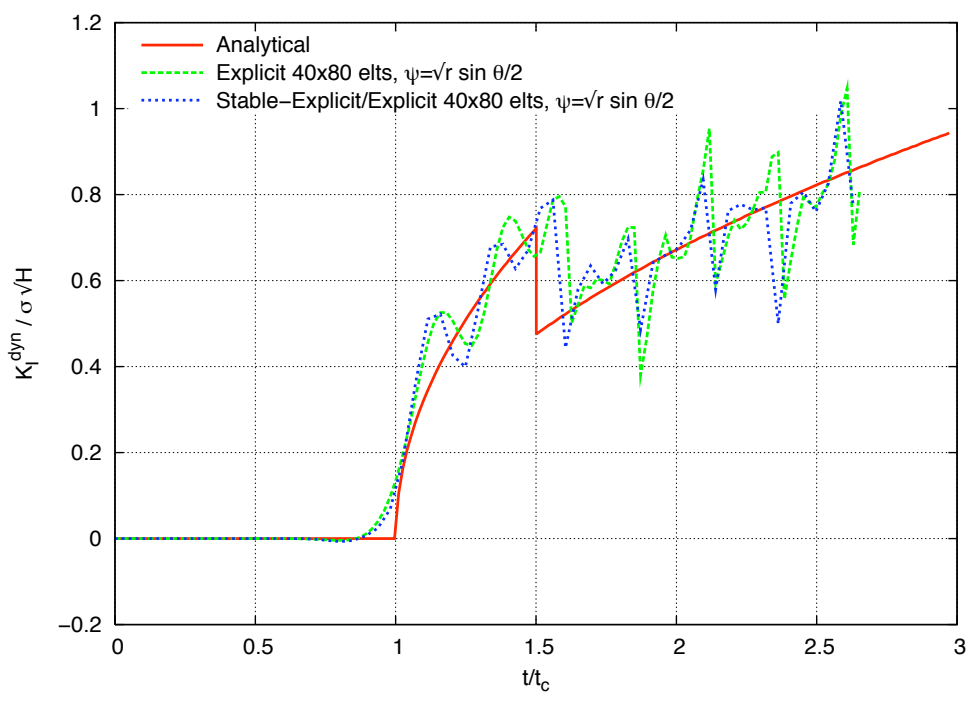

Fig. 7. Normalized mode I stress intensity factor vs. normalized time for a stationary then moving semi-infinite crack: analytical solution, $40 \times 80$ elements mesh with central difference explicit scheme and time step rule of Part $1\left(\Delta t=\Delta t_{c} / 2\right), 40 \times 80$ elements mesh with element-by-element explicit scheme $\left(\Delta t=\Delta t_{c}\right)$.

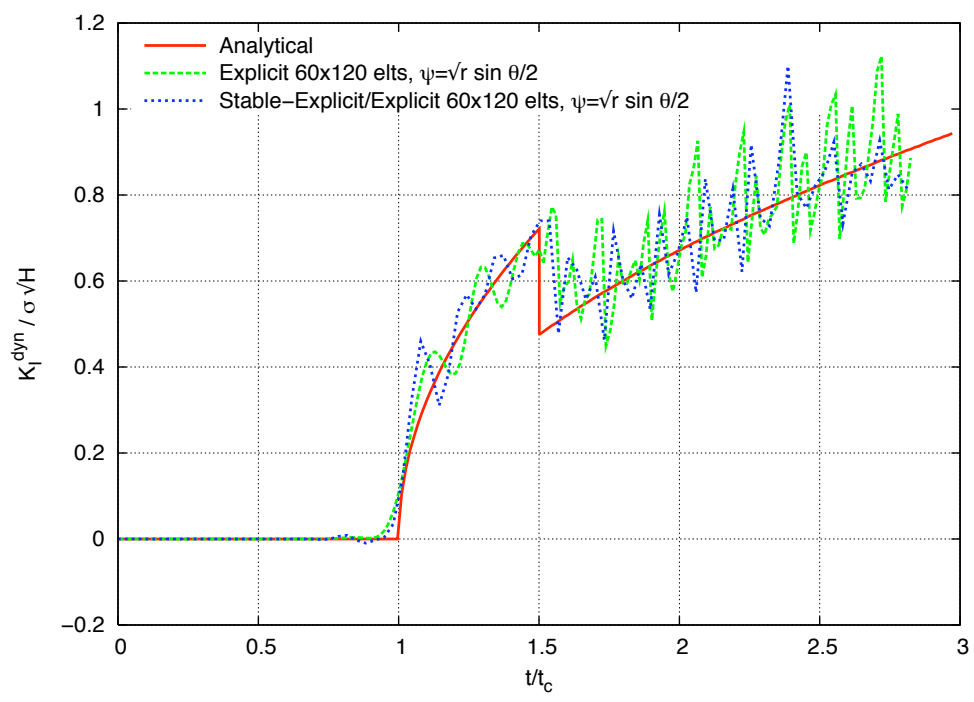

Fig. 8. Normalized mode I stress intensity factor vs. normalized time for a stationary then moving semi-infinite crack: analytical solution, $60 \times 120$ elements mesh with central difference explicit scheme and time step rule of Part $1\left(\Delta t=\Delta t_{c} / 2\right), 60 \times 120$ elements mesh with element-by-element explicit scheme $\left(\Delta t=\Delta t_{c}\right)$.

indicates that the accuracy is increased by using the proposed method.

In Figure 8, we compare the results obtained with a mesh composed of $60 \times 120$ quadrilateral elements with a standard explicit central difference scheme with a critical time step of $\Delta t_{c}^{x-f e m}=5 \mu s \simeq \Delta t_{c}^{f e m} / 2$ (that is a simulation of 200 time steps) and the proposed Stable-Explicit/Explicit scheme with a critical time step of $\Delta t_{c}^{x-f e m}=\Delta t_{c}^{f e m}=10.99 \mu s$ (that is a simulation of 91 time steps). 
As observed previously, both results compare well to the analytical solution and the proposed method has fewer oscillations with a lower amplitude. It can be noted that for both methods, the oscillations are lower compared to the results obtained with the coarser mesh.

\subsection{Kalthoff's experiment}

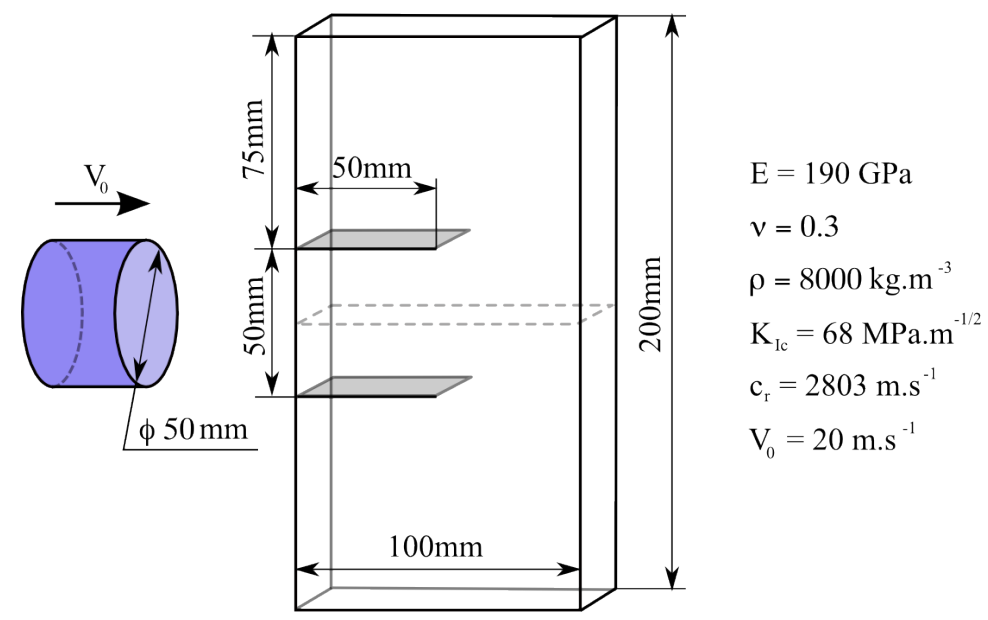

Fig. 9. Experimental setup and geometry for Kalthoff's experiment.

This example deals with the numerical simulation of Kalthoff's experiment of the failure mode transition under pure mode II loading [26]. The experimental configuration is presented in Figure 9: a plate with two symmetrical edge cracks is impacted by a projectile at a given speed $V_{0}$. By modifying the projectile's velocity, Kalthoff observed a transition in the type of failure. At low velocity, i.e. under low strain rate, brittle failure is observed with a simultaneous propagation of the two cracks with a global angle between $60^{\circ}$ and $70^{\circ}$. If one increases the projectile's speed, a transition between brittle fracture and shear band propagation (with a propagation angle of approximatively $-10^{\circ}$ ) occurs. We consider here only the brittle propagation with in impact velocity of $20 \mathrm{~m} . \mathrm{s}^{-1}$. As in Belytschko et al. [4], Zi et al. [42] and Menouillard et al. [29], we model the experiment in plain strain and consider only the upper half of the plate with the appropriate symmetry boundary conditions. The material properties are those of a 18Ni1900 maraging type steel and are given in Figure 9.

A mesh of $80 \times 80$ piecewise bilinear quadrilateral elements was used, with a critical time step of $\Delta t_{c}^{x-f e m}=\Delta t_{c}^{f e m} / 2=0.11 \mu s$ for the explicit central difference scheme, the simulation time was $100 \mu s$ represented by 900 time steps. When using the proposed Stable-Explicit/Explicit time scheme, a critical time step of $\Delta t_{c}^{x-f e m}=\Delta t_{c}^{f e m}=0.22 \mu s$, the simulation time was $100 \mu s$ represented by 450 time steps. 

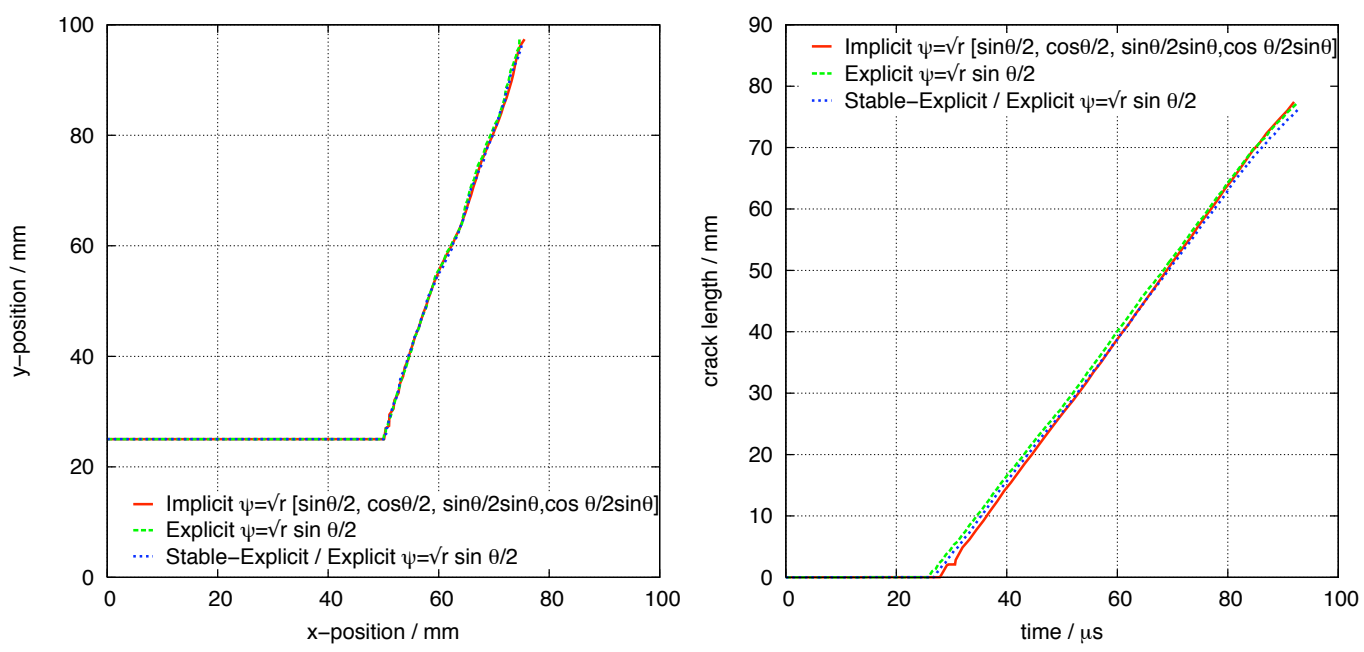

Fig. 10. Kalthoff's experiment, results for the proposed method with the element-by-element explicit scheme $\left(\Delta t=\Delta t_{c}\right)$, with central difference scheme and the time step rule of Part $1\left(\Delta t=\Delta t_{c} / 2\right)$ and for an implicit X-FEM calculation (from [29]): final crack path (left) and crack length versus time (right).

The left part of Figure 10 shows the crack path obtained with and without the proposed explicit dynamic scheme and with an X-FEM implicit mean acceleration time integrator with a full singular enrichment basis with the same mesh (Menouillard et al. [29]). These results are very similar and the overall angle is in good agreement with the experimental and previously published numerical results. Looking at the details of the crack path, we observe the same phenomena as in Menouillard et al. [29] and de Borst et al. [16]. The crack starts to propagate with an angle of $65^{\circ}$ at $26 \mu \mathrm{s}$; then a small deviation is observed around $50 \mu \mathrm{s}$ and the crack continues to propagate with an angle of $65^{\circ}$ from $65 \mu \mathrm{s}$. This deviation is related to the successive reflections of the compressive stress wave. The wave travels from the left edge to the right one when the projectile hits the plate. Then the wave is reflected on the right free surface as a tensile wave, reaches the crack tip around $26 \mu s$ and the propagation starts. This tensile wave travels from the crack tip to the left free surface and is again reflected as a compressive wave. Then, this wave travels in the right direction and reaches the moving crack tip around $50 \mu \mathrm{s}$. This compressive wave induces the crack deviation, but is subsequently reflected against the right edge as a tensile wave which finally reaches the crack tip at $65 \mu s$, after which the crack continues to propagate with the initial angle.

We also plot on the right part of Figure 10 the crack length as a function of time for the two methods. We can also observe that the three results are almost identical, although the crack seams to propagate a little earlier and with a slightly lower velocity with the explicit schemes than with the implicit one.

This results show that the proposed explicit dynamic scheme allows us to 
obtain the same results obtained in Part 1 with a reduced computational cost as the number of time step is divided by a factor of two.

\subsection{Grégoire et al.'s experiment}

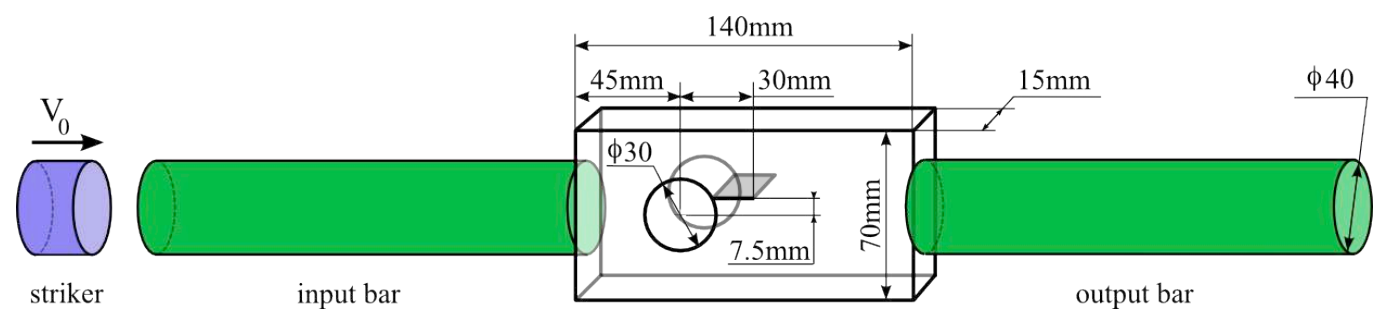

Fig. 11. Experimental setup and geometry for Grégoire's experiment.

The last example is based on the experiment of Grégoire et al. proposed in [22]. The experimental setup can be observed in Figure 11. It consists of a Split Hopkinson Pressure Bar test rig. The test specimen is made out of PMMA and consists in a rectangular plate with a hole and a initial notch coming out of the hole. The hole provides a direct conversion of compressive waves coming from the impact of the input bar into tensile waves. In order to ensure mixed-mode loading and crack orientation effects during the propagation, the initial notch is moved upwards from the specimen axis of symmetry. Strain gages are placed on the input and output bars. The measured signal can be interpreted in term of strength or velocity. An optical measurement system composed of four digital cameras is used to take pictures during the test and obtain precise information on the crack position.

This experiment is composed of three phases: the crack initiates at around $200 \mu \mathrm{s}$ and propagates at a constant horizontal velocity of approximatively $\dot{a}_{x} \simeq 211 \mathrm{~m} . \mathrm{s}^{-1}$ which corresponds to a maximum of the curvilinear velocity of the crack tip $\dot{a} \simeq 260 \mathrm{~m} . \mathrm{s}^{-1}$; then the crack stops for about $50 \mu \mathrm{s}$; finally a second propagation stage at constant horizontal velocity $\dot{a}_{x} \simeq 157 \mathrm{~m} . \mathrm{s}^{-1}$ $\left(\dot{a} \simeq 160 \mathrm{~m} . \mathrm{s}^{-1}\right)$ occurs until the final stop at about $500 \mu \mathrm{s}$.

As in Grégoire et al. [22] the hole and the crack are modelled using enrichment. The Stable-Explicit element group is therefore composed of the elements enriched to represent the crack and elements cut by the hole. We used a regular mesh of $27 \times 51$ piecewise bilinear quadrilateral elements. The experimental velocity is imposed as the loading on the contact face between the input bar and the specimen. On the contact surface between the specimen and the output bar, an impedance boundary condition is used to model the output bar without spurious wave reflections. The impedance conditions consists in imposing a $1 \mathrm{D}$ relation between the horizontal component of the stress vector 
and the horizontal velocity on the surface considered. This can be written as:

$$
\boldsymbol{\sigma} \cdot \mathbf{n}=-z(\dot{\mathbf{u}} \cdot \mathbf{n}) \mathbf{n}
$$

where $z$ is related to the material properties of the output bar by the following

$$
z=\rho^{b a r} c_{L}^{b a r}
$$

$\rho^{b a r}$ and $c_{L}^{b a r}$ being respectively the ouput bar density and unidimensional traction-compression wave speed. The integration of this $1 \mathrm{D}$ condition results in a additional force term in the equilibrium equation:

$$
M \ddot{U}_{n+1}=F_{n+1}-F_{n+1}^{i n t}-Z \dot{U}_{n+1}
$$

which can be simplified using the update equations of the time integration scheme:

$$
(M+\Delta t Z) \ddot{U}_{n+1}=F_{n+1}-F_{n+1}^{i n t}-Z\left(\dot{U}_{n}+\Delta t \ddot{U}_{n}\right) .
$$

The matrix $Z$ that comes from the integration of Eq. (35) is diagonal, which allows us to easily solve Eq. (38), the global matrix being still diagonal.

The material properties ar given in Table 1. As in Grégoire et al. [22], we use two different values for the dynamic fracture toughness: one for the initiation and one for the propagation. This is due in part to the radius difference between the initial notch and the propagating crack.

\begin{tabular}{cc}
\hline Young's modulus $E$ & $2.4 \mathrm{GPa}$ \\
Poisson ratio $\nu$ & 0.42 \\
Density $\rho$ & $1180 \mathrm{~kg} \cdot \mathrm{m}^{-3}$ \\
Dilatational wave speed $c_{L}$ & $1426 \mathrm{~m} . \mathrm{s}^{-1}$ \\
Shear wave speed $c_{S}$ & $846 \mathrm{~m} . \mathrm{s}^{-1}$ \\
Rayleigh wave speed $c_{R}$ & $800 \mathrm{~m} . \mathrm{s}^{-1}$ \\
Initiation fracture toughness $K_{I c}^{\text {ini }}$ & $1.47 \mathrm{MPa} \cdot \sqrt{\mathrm{m}}$ \\
Propagation fracture toughness $K_{I c}^{\text {propa }}$ & $1.33 \mathrm{MPa} \cdot \sqrt{\mathrm{m}}$ \\
\hline
\end{tabular}

Table 1

Grégoire's experiment: material properties.

The results of the computation can be seen in Figure 12 to 15. In Figure 12, we plot the crack tip horizontal position as a function of time for the simulation and the experiment. We can see that the initiation, arrest and restart phases are well captured as it is the case in Grégoire et al. [22] with an X-FEM implicit mean acceleration calculation. In particular the arrest phase position in space and time is well described compared to the results obtained in Prabel et al. [31]. We can note however that the initiation occurs a little earlier than in the experiment and that the crack velocity in both propagation phases is 


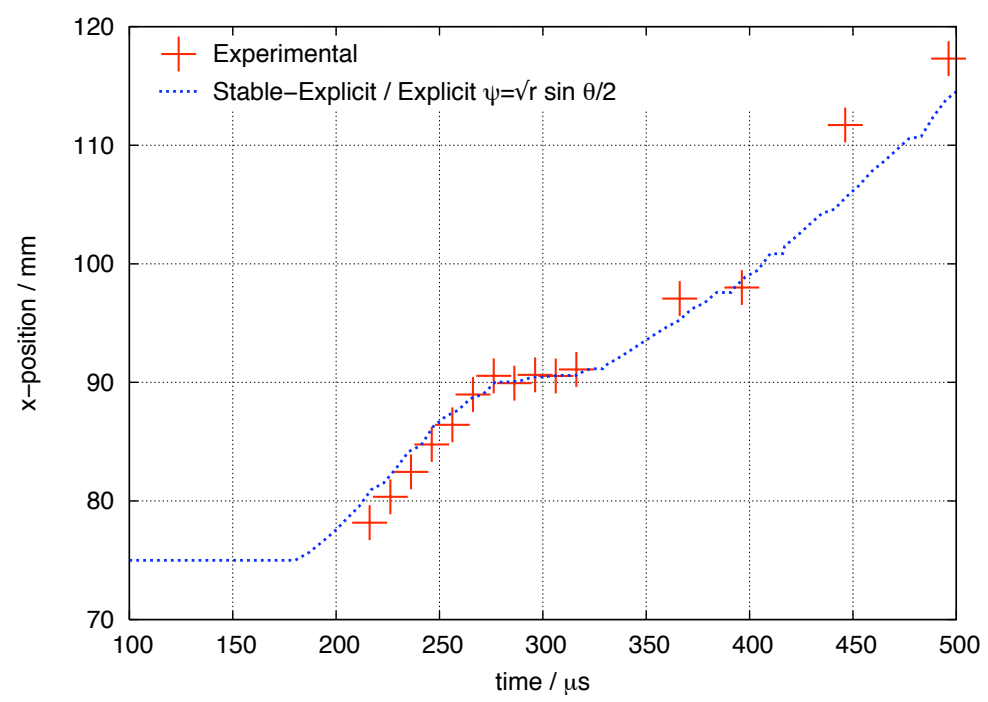

Fig. 12. Grégoire's experiment: results from the experiment (from [22]) and the numerical simulation with the proposed method: crack tip abscissa versus time.

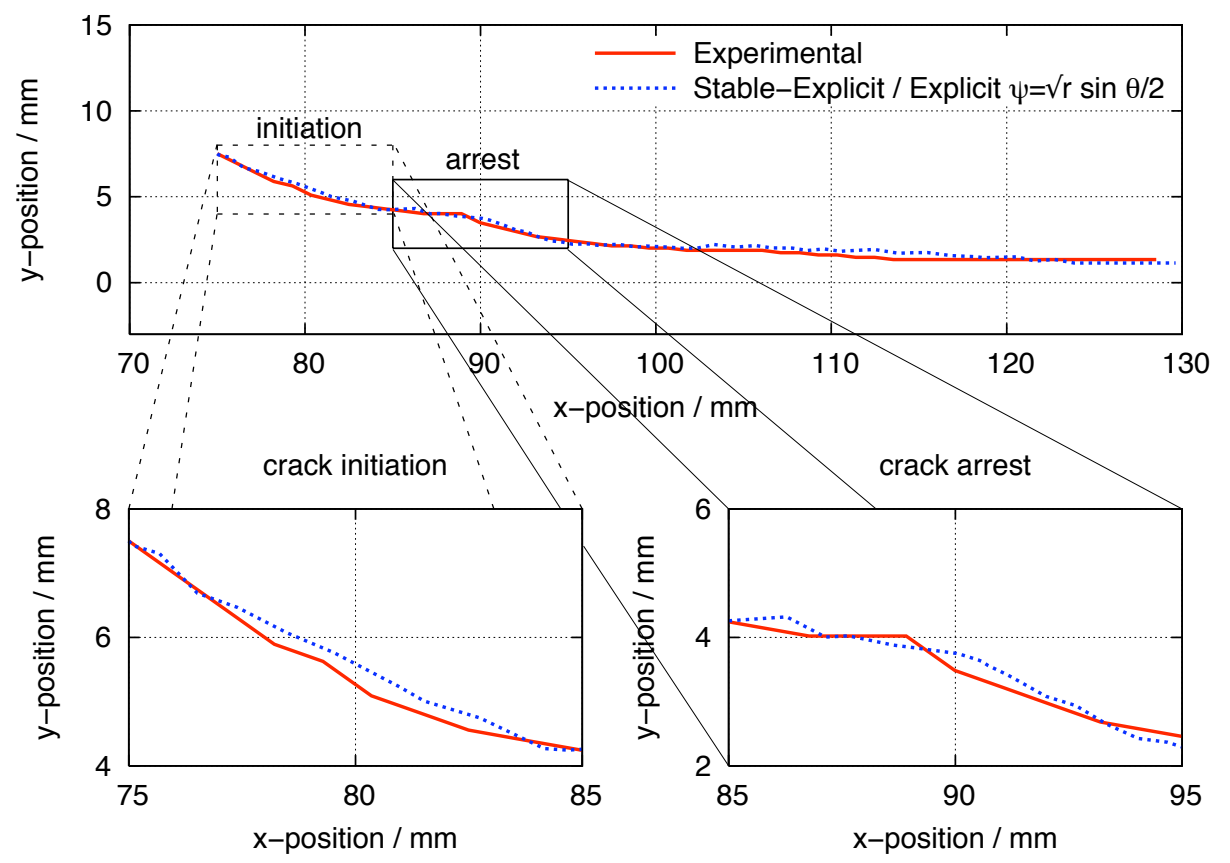

Fig. 13. Grégoire's experiment: results from the experiment (from [22]) and the numerical simulation with the proposed method: global crack path and zoomed crack path around initiation and arrest. 
slightly lower than the experimental one. We can also note that there is no spurious crack kinking in the last propagation phase compared to the results of Prabel et al. [31].

In Figure 13, we compare the experimental and numerical crack paths. We can observe the global path obtained in the calculation agrees well with the experimental one. A zoom on the results around the initiation and arrest phases confirms this agreement in these important zones of the crack path.

In Figure 14, we plot the input and output experimental and numerical velocities as a function of time. We can see that the numerical output velocity presents some oscillations but agrees quite well with the experimental one. However, we can see that there is a shift in time of around $20 \mu \mathrm{s}$ on the output velocity which corresponds to the difference in the initiation time between the simulation and the experiment. This indicates that some improvements can be done in the method to fit even better the experimental results.

Finally we plot on Figure 15 the vertical displacement field on the deformed configuration (amplified 1.5 times) and the Von Mises stress field on the initial configuration at various interesting times.

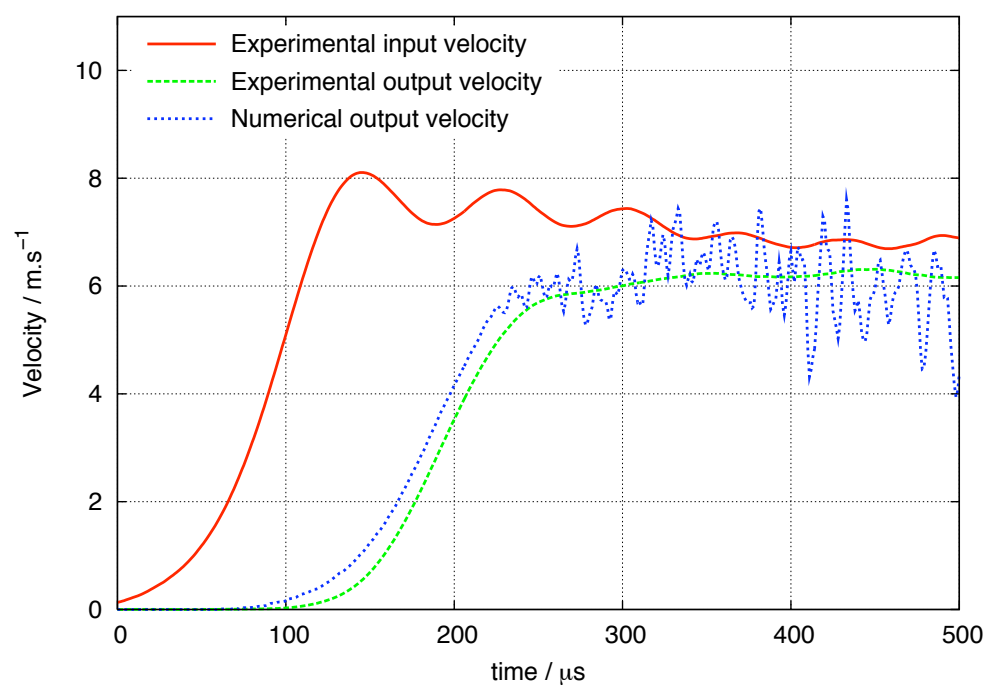

Fig. 14. Grégoire's experiment: results from the experiment (from [22]) and the numerical simulation with the proposed method: input and output velocities versus time. 

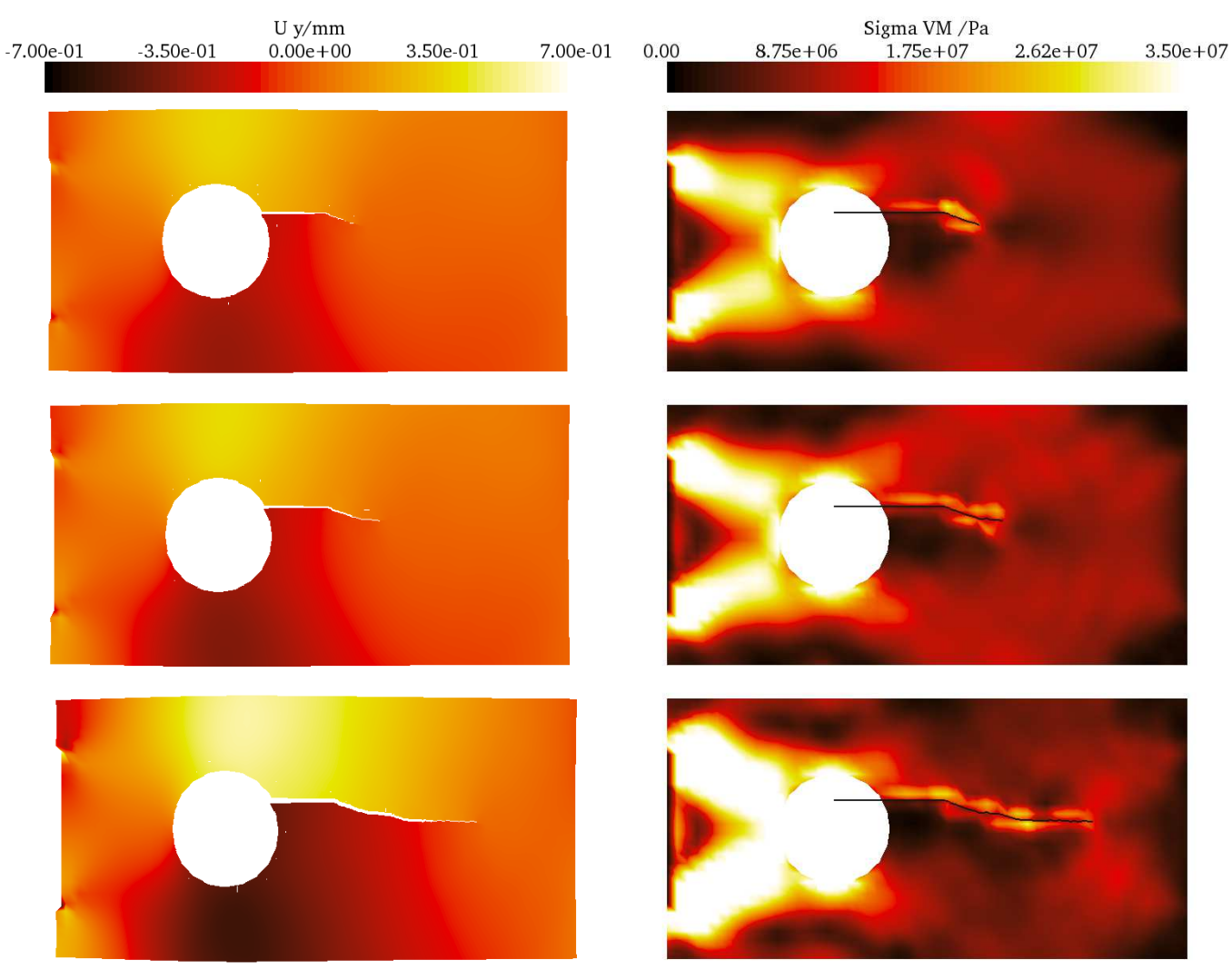

Fig. 15. Grégoire's experiment: results at $235 \mu \mathrm{s}, 320 \mu \mathrm{s}$ and $500 \mu \mathrm{s}$. Left: vertical displacement on the deformed configuration (amplified 1.5 times), right: Von Mises stress field on the initial configuration (the crack is represented by the black segments).

\section{Conclusions}

We have proposed in this paper a combination of a general mass lumping technique for arbitrary enrichments with a stable explicit-explicit strategy dedicated to X-FEM. In a general point of view, it allows to recover a standard critical time step in the X-FEM framework for fully explicit transient dynamic simulations with possible moving interfaces. Indeed, no enrichment dependency of the critical time step is observed, i.e. the position of the interface with respect to the nodes and element boundaries does not decreases the value of the critical time step. For that purpose, an unconditionally stable explicit time scheme has been coupled with the central difference method in an element-by-element partitioning. Furthermore, a study of the global stability properties has been proposed via the energy method. The interest of the combination of the mass lumping formula presented in Part 1 and the element-by-elements scheme is that a complex structure can be modelled by a rectangular structured mesh, the inside and outside complex shapes being modelled with hole enrichment. With such an approach, as presented in the 
numerical examples, the critical time step determination is very easy and the number of time steps is minimal while accuracy is maximum.

The application of the proposed method to dynamic crack growth in mixed mode reveals its ability to simulate moving interfaces in an fully explicit XFEM framework with a standard critical time step. In this particular case, It can be noticed that asymptotic enrichments with moving crack has been used here for the first time with an explicit X-FEM strategy. This is of great importance for future application such as tridimensional dynamic crack growth simulations. Indeed, in such a case, a very good accuracy is required along the crack front, both in term of shape modeling, and also in term of discontinuous and asymptotic behavior along the crack front. In a next step, extensions of such an approach to non-linear behavior, both in the bulk or inside the interface will be considered in an explicit X-FEM framework. This is of great interest for transient highly nonlinear dynamics simulations with a large number of time step which also require to take into account moving interfaces.

\section{Acknowledgements}

This work was supported by the French Agence Nationale pour la Recherche and Centre National de la Recherche Scientifique under contract ANR-CNRS DYNRUPT JC05_45254. This support is gratefully acknowledged. We would also like to thank Dr Grégoire for providing results for the last example.

\section{References}

[1] I. Babuška and J.M. Melenk. The partition of unity method. International Journal for Numerical Methods in Engineering, 40:727-758, 1997.

[2] K.J. Bathe and E.L. Wilson. Stability and accuracy analysis of direct integration methods. Earthquake Engineering and Structural Dynamics, 1:238-291, 1973.

[3] T. Belytschko and H. Chen. Singular enrichment finite element method for elastodynamic crack propagation. International Journal of Computational Methods, 1:1-15, 2004.

[4] T. Belytschko, H. Chen, J. Xu, and G. Zi. Dynamic crack propagation based on loss of hyperbolicity and a new discontinuous enrichment. International Journal for Numerical Methods in Engineering, 58:1873-1905, 2003.

[5] T. Belytschko and R. Mullen. Stability of explicit-implicit mesh partitions in time integration. International Journal for Numerical Methods in Engineering, 12(10):1575-1586, 1978. 
[6] T. Belytschko and D.F. Schoeberle. On the unconditional stability of an implicit algorithm for nonlinear structural dynamics. Journal of Applied Mechanics, 17:865-869, 1975.

[7] T. Belytschko and P. Smolinski. Stability of multi-time step partitioned integrators for first-order finite element systems. Computer Methods in Applied Mechanics and Engineering, 49(3):281-297, 1985.

[8] T. Belytschko, H.J. Yen, and R. Mullen. Mixed methods for time integration. Computer Methods in Applied Mechanics and Engineering, 17(18):259-275, 1979.

[9] S.Y. Chang. Explicit pseudodynamic algorithm with unconditional stability. Journal of Engineering Mechanics, 128:935-947, 2002.

[10] S.Y. Chang. Enhanced, unconditionally stable, explicit pseudodynamic algorithm. Journal of Engineering Mechanics, 133:541-554, 2007.

[11] S.Y. Chang. An explicit method with improved stability property. International Journal for Numerical Methods in Engineering, 77(8):1100-1120, 2008.

[12] A. Combescure and A. Gravouil. A numerical scheme to couple subdomains with different time-steps for predominantly linear transient analysis. Computer Methods in Applied Mechanics and Engineering, 191(11):1129-1157, 2002.

[13] A. Combescure, A. Gravouil, D. Grégoire, and J. Réthoré. X-FEM a good candidate for energy conservation in simulation of brittle dynamic crack propagation. Computer Methods in Applied Mechanics and Engineering, 197(5):309-318, 2008.

[14] R. Courant, K. Friedrichs, and H. Lewy. Uber die partiellen differenzengleichungen der mathematischen physik. Mathematische Annalen, 100:32-74, 1928.

[15] W.J.T. Daniel. A study of the stability of subcycling algorithms in structural dynamics. Computer Methods in Applied Mechanics and Engineering, 156(1-4):1-13, 1998.

[16] R. de Borst, J.J.C. Remmers, and A. Needleman. Mesh independant discrete numerical representations of cohesive-zone models. Engineering fracture mechanics, 73(2):160-177, 2006.

[17] C.A. Duarte, O.N. Hamzeh, T.J. Liszka, and W.W. Tworzydlo. A generalized finite element method for the simulation of three-dimensional dynamic crack propagation. Computer Methods in Applied Mechanics and Engineering, 190:2227-2262, 2000.

[18] T. Elguedj, A. Gravouil, and H. Maigre. An explicit dynamics extended finite element method. Part 1: Mass lumping for arbitrary enrichment functions. Computer Methods in Applied Mechanics and Engineering, 2008, submitted.

[19] L.B. Freund. Dynamic Fracture Mechanics. Cambridge University Press, Cambridge, UK, 1990.

[20] G.L. Goudreau and R.L. Taylor. Evaluation of numerical integration methods in elastodynamics. Computer Methods in Applied Mechanics 
and Engineering, 2:69-97, 1972.

[21] A. Gravouil and A. Combescure. Multi-time-step explicit-implicit method for non-linear structural dynamics. International Journal for Numerical Methods in Engineering, 50:199-225, 2001.

[22] D. Grégoire, H. Maigre, J. Réthoré, and A. Combescure. Dynamic crack propagation under mixed-mode loading - Comparison between experiments and X-FEM simulations. International Journal of Solids and Structures, 44:6517-6534, 2007.

[23] H.M. Hilber and T.J.R. Hughes. Collocation, dissipation and overshoot for time integration schemes in structural dynamics. Earthquake Engineering and Structural Dynamics, 6:99-117, 1978.

[24] T.J.R. Hughes. The Finite Element Method: Linear Static and Dynamic Finite Element Analysis. Dover Publications, Mineola NY, 2000.

[25] T.J.R. Hughes and W.K. Liu. Implicit-explicit finite elements in transient analysis: implementation and numerical examples. Journal of Applied Mechanics, 45:375-378, 1978.

[26] J.F. Kalthoff. Modes of dynamic shear failure in solids. International Journal of Fracture, 101:1-31, 2000.

[27] R.D. Krieg and S.W. Key. Transient shell response by numerical time integration. International Journal for Numerical Methods in Engineering, 7:273-286, 1973.

[28] T. Menouillard. Dynamique explicite pour la simulation numérique de propagation de fissure par la méthode des éléments finis étendus (in French). PhD thesis, Institut National des Sciences Appliquées de Lyon, France, 2007.

[29] T. Menouillard, J. Réthoré, A. Combescure, and H. Bung. Efficient explicit time stepping for the extended finite element method. International Journal for Numerical Methods in Engineering, 68:911-938, 2006.

[30] T. Menouillard, J. Réthoré, N. Moës, A. Combescure, and H. Bung. Mass lumping strategies for X-FEM explicit dynamics: Application to crack propagation. International Journal for Numerical Methods in Engineering, 74(3):447-474, 2008.

[31] B. Prabel, A. Combescure, A. Gravouil, and S. Marie. Level set XFEM non matching meshes: Application to dynamic crack propagation in elastic-plastic media. International Journal for Numerical Methods in Engineering, 69:1553-1569, 2007.

[32] J.J.C. Remmers, R. de Borst, and A. Needleman. The simulation of dynamic crack propagation using the cohesive segments method. Journal of the Mechanics and Physics of Solids, 56:70-92, 2008.

[33] J. Réthoré, A. Gravouil, and A. Combescure. A combined space-time extended finite element method. International Journal for Numerical Methods in Engineering, 64:260-284, 2005.

[34] J. Réthoré, A. Gravouil, and A. Combescure. An energy-conserving scheme for dynamic crack growth using the extended finite element method. International Journal for Numerical Methods in Engineering, 
63:631-659, 2005.

[35] P. Rozycki, N. Moës, E. Béchet, and C. Dubois. X-FEM explicit dynamics for contant strain elements to alleviate mesh constraints on internal or external boundaries. Computer Methods in Applied Mechanics and Engineering, 197:349-363, 2008.

[36] D. Sha, K.K. Tamma, and X. Zhou. Time discretized operators. Part 2: towards the theoretical design of a new generation of a generalized family of unconditionally stable implicit and explicit representations of arbitrary order for computational dynamics. Computer Methods in Applied Mechanics and Engineering, 192:291-239, 2003.

[37] P. Smolinski, T. Belytschko, and M. Neal. Multi-time-step integration using nodal partitioning. International Journal for Numerical Methods in Engineering, 26:349-359, 1988.

[38] P. Smolinski and Y.S. Wu. Stability of explicit subcycling time integration with linear interpolation for first-order finite element semidiscretizations. Computer Methods in Applied Mechanics and Engineering, 151(3-4):311324, 1998.

[39] D.E. Sotelino. A concurrent explicit-implicit algorithm in structural dynamics. Computers and Structures, 51(2):181-190, 1994.

[40] K.K. Tamma, D. Sha, and X. Zhou. Time discretized operators. Part 1: towards the theoretical design of a new generation of a generalized family of unconditionally stable implicit and explicit representations of arbitrary order for computational dynamics. Computer Methods in Applied Mechanics and Engineering, 192:257-290, 2003.

[41] X. Zhou, D. Sha, and K.K. Tamma. A novel non-linearly explicit second-order accurate L-stable methodology for finite deformation: hypoelastic/hypoelasto-plastic structural dynamics problems. International Journal for Numerical Methods in Engineering, 59:795-823, 2004.

[42] G. Zi, H. Chen, J. Xu, and T. Belytschko. The extended finite element method for dynamic fractures. Shock and Vibration, 12:9-23, 2005. 